\title{
Surface effect on the resonant frequency of Timoshenko nanobeams
}

\author{
Ning Jia ${ }^{\mathrm{a}, \mathrm{e}}$, Yin Yao ${ }^{\mathrm{b}, \mathrm{c}}$, Yazheng Yang ${ }^{\mathrm{b}, \mathrm{c}, \mathrm{d}}$, Shaohua Chen ${ }^{\mathrm{b}, \mathrm{c}, \mathrm{d}, *}$ \\ ${ }^{a}$ LNM, Institute of Mechanics, Chinese Academy of Sciences, Beijing 100190, China \\ ${ }^{\mathrm{b}}$ Institute of Advanced Structure Technology, Beijing Institute of Technology, Beijing 100081, China \\ c Beijing Key Laboratory of Lightweight Multi-functional Composite Materials and Structures, Beijing Institute of Technology, Beijing 100081, China \\ ${ }^{\mathrm{d}}$ Collaborative Innovation Center of Electric Vehicles in Beijing, Beijing Institute of Technology, Beijing 100081, China \\ e School of Engineering Sciences, University of Chinese Academy of Sciences, Beijing 100049, China
}

\section{A R T I C L E I N F O}

\section{Keywords:}

Timoshenko nanobeam

Resonant frequency

Surface effect

Shear deformation effect

Rotary inertia effect

\begin{abstract}
A B S T R A C T
The dynamic behavior of a Timoshenko nanobeam would be significantly different from a macro-one due to the large ratio of surface area to volume of nanomaterials. Furthermore, the shear deformation effect would be obvious for a Timoshenko nanobeam in contrast to an Eulerian one. In this paper, a recently developed elastic theory is adopted in order to predict the resonant frequency of a Timoshenko nanobeam, in which not only the surface effect but also the shear deformation effect and the rotary inertia one are considered. In contrast to the existing surface effect theories, surface effect of nanomaterials is characterized by the surface energy density in the adopted theory. The resonant frequency of both a fixed-fixed nanobeam and a cantilevered one is analyzed. It is found that the dynamic behavior of nanobeams deviates significantly from the one predicted by both the classical Timoshenko beam theory and the Euler-Bernoulli one due to the surface effect. Furthermore, the shear deformation effect and the rotary inertia effect cannot be neglected in nanobeams with a relative small aspect ratio, which cannot be precisely characterized by the Euler-Bernoulli beam theory. In addition, the influencing mechanism of surface effect on the dynamic behavior of nanobeams would depend on the boundary conditions. The resonant frequency of a fixed-fixed Timoshenko nanobeam would be improved, while that of a cantilevered one would be weakened by the surface effect in contrast to the corresponding classical solutions. The results in this paper should be useful for precise design of nano-devices and helpful for reasonable assessment of test results of nano-instruments.
\end{abstract}

(C) 2017 Elsevier Ltd. All rights reserved.

\section{Introduction}

Nanobeam, as a simple but promising elements, has been widely used in flexible electronics [1], biological sensors [2] and nano-electromechanical systems (NEMS) [3]. There is no doubt that its static and dynamic mechanical behaviors attract much attention. Many experiments have shown that not only the static mechanical properties but also the dynamic ones of nanobeams are significantly different from those of macro-beams due to the large surface-to-volume ratio of nanomaterials, such as the effective Young's modulus and resonant frequency [4-12].

Compared with the static bending experiment, resonance measurement is a more convenient treatment to achieve nanobeams' elastic properties in view of the clear relationship between the resonant frequency and elastic parameters of a beam [13-15]. The effective Young's moduli of silicon, gallium nitride $(\mathrm{GaN})$, silicon nitride $\left(\mathrm{SiN}_{\mathrm{x}}\right)$ and zinc oxide $(\mathrm{ZnO})$ cantilever nanowires could be achieved experimentally by measuring the resonant frequency of vibrating nanobeams $[4-7,11]$. However, classical beam theories are invalid for predicting the vibration properties of nanobeams, due to the lack of parameters accounting for the surface effect in nanomaterials. Several researchers proposed strain gradient elasticity theories and nonlocal elasticity theory with intrinsic length scales to study the size-dependent mechanical behaviors of microor nano-scale beams [16-22], but how to interpret and determine the intrinsic lengths is still a challenging issue in these high-order theories.

The surface elasticity theory established by Gurtin and Murdoch $[23,24]$ (G-M model) were widely adopted recently, instead of the classical continuum one to study the mechanical behaviors of nanomaterials and nanostructures, in which the surface is assumed to have a zerothickness and abide by the elastic constitutive relation. Based on the G-M model and its extension, not only the elastic behavior of staticbending nanobeams but also the dynamic vibration property was carried out [13,14,20,25-31]. Both the effective Young's modulus and the resonant frequency of nanobeams depend on the surface elastic modulus. It was further found that the mechanical parameters are also influenced by the boundary conditions. As for the dynamic vibration behav-

\footnotetext{
* Corresponding author at: Beijing Institute of Technology, Beijing 100081, China

E-mail addresses: chenshaohua72@hotmail.com, shchen@bit.edu.cn (S. Chen).
} 
ior of nanobeams, the normalized resonant frequency of a fixed-fixed nanobeam (both ends fixed) would increase, while that of a cantilevered nanobeam decreases with a reducing characteristic size. Chiu and Chen [32] further discussed the effect of the surface bending modulus on the resonant frequency of nanowires. Recently, Zhang et al. [33] analyzed the motion equation of vibrating nanowires, based on which contributions of surface elasticity and surface stress to the resonant frequency can be abstracted, respectively.

Though the surface elasticity theory is a recently popular theoretical approach in predicting the mechanical properties of nanomaterials and nanostructures, the surface elastic constants impose some restrictions on its applications. The surface elastic constants are introduced in order to describe the elastic constitutive relation between the surface stress and surface strain. However, it is found that the surface elastic constants cannot be measured experimentally till now. The unique technique to find the parameters is MD simulations [34-36]. Even in MD calculations, some problems still exist, such as how to choose a proper atomic potential, how to choose the numerical model size and how many atom layers could be regarded as the surface of a nanobeam. Therefore, to determine surface elastic constants in the surface elasticity theory is a key problem, which is worth focusing on in the future.

In view of the above thorny problem, an alternative elastic theory has been established for nanomaterials by Chen and Yao [37] within the framework of continuum mechanics. The surface energy density is used to characterize the surface effect in nanomaterials instead of the introduction of surface elastic constants, which depends only on the bulk surface-energy density and the surface-relaxation parameter. Both parameters are easily found from Material Handbooks or simple MD simulations. Typical problems have been well analyzed based on such a novel theory and the predicted results agree well with the existing experimental data and numerical calculations [38-41].

In this paper, the developed theory [37] will be adopted to analyze the resonant vibration of Timoshenko nanobeams. Not only the surface effect but also the shear deformation effect and the rotary inertia one should be included [26-28,42]. Comparison of theoretical predictions of Euler-Bernoulli nanobeams and Timoshenko ones as well as the classical solutions of Timoshenko macro-beams will be carried out. Respective influence ranges of the surface effect, the shear deformation and rotary inertia effect on the resonant frequency of beams will be given, which should be helpful for the design of beam-based nano-devices, in especial, the nanobeam resonators and sensors in NEMS.

\section{A brief introduction of the surface energy density-based elastic theory for nanomaterials}

Based on the concept of surface energy density, an elastic theory was developed by Chen and Yao [37] in order to study the surface effect in nanomaterials or nanostructures. With a similar idea, an interface energy density-based theory was also established in order to investigate the interface effect in nanocomposites [43]. The elastic theory with surface effect is briefly introduced in this section [37].

The governing equation inside a nano-solid and the boundary conditions at the surface are written as

$$
\left\{\begin{array}{l}
\boldsymbol{\sigma} \cdot \nabla+\boldsymbol{f}=0 \quad \text { (in } V-S) \\
\boldsymbol{n} \cdot \boldsymbol{\sigma} \cdot \boldsymbol{n}=\boldsymbol{p} \cdot \boldsymbol{n}-\gamma_{n} \boldsymbol{n} \quad(\text { on } S) \\
(\boldsymbol{I}-\boldsymbol{n} \otimes \boldsymbol{n}) \cdot \boldsymbol{\sigma} \cdot \boldsymbol{n}=(\boldsymbol{I}-\boldsymbol{n} \otimes \boldsymbol{n}) \cdot p-\gamma_{t} \quad(\text { on } S)
\end{array}\right.
$$

where $\sigma$ is the Cauchy stress tensor inside the nano-solid, $\nabla$ denotes a gradient operator. $\mathbf{n}$ is the unit normal vector perpendicular to the boundary surface $S$ of the nano-solid. I is a unit tensor; $\mathbf{f}$ and $\boldsymbol{p}$ denote the body force and the external surface traction, respectively. $V$ is the volume of the nano-solid. $\gamma_{n}$ is the normal component and $\gamma_{t}$ is the tangential one of an additionally surface-induced traction vector, respectively, which amounts to a force disturbance at the boundary due to the surface effect and can be obtained with the help of the virtual work method based on an infinitesimal element,

$\gamma_{t}=\nabla_{s} \phi, \gamma_{n} \mathbf{n}=\phi\left(\frac{1}{R_{1}}+\frac{1}{R_{2}}\right) \mathbf{n}=\phi\left(\mathbf{n} \cdot \nabla_{s}\right) \mathbf{n}$

where $\nabla_{s}$ denotes a surface gradient operator. $\phi$ is the surface energy density of the nano-solid in the current configuration, which is called as Eulerian surface energy density (relative to the initial or reference configuration as shown in Fig. 1). $R_{1}$ and $R_{2}$ are two principal curvature radii of a curved surface.

The relation between the Eulerian surface energy density $\phi$ in the current configuration and the Lagrangian surface energy density $\phi_{0}$ in the reference configuration is

$\phi=\frac{\phi_{0}}{J_{s}}$

where $J_{s}$ is a Jacobean determinant characterizing the surface deformation from the reference configuration to the current one.

Combining Eqs. (1)-(3) yields the final governing equation and boundary conditions

$\left\{\begin{array}{l}\boldsymbol{\sigma} \cdot \nabla+\boldsymbol{f}=0 \quad(\text { in } V-S) \\ \boldsymbol{n} \cdot \boldsymbol{\sigma} \cdot \boldsymbol{n}=\boldsymbol{p} \cdot \boldsymbol{n}-\phi_{0}\left(\boldsymbol{n} \cdot \nabla_{s}\right) / J_{s} \quad(\text { on } S) \\ (\boldsymbol{I}-\boldsymbol{n} \otimes \boldsymbol{n}) \cdot \boldsymbol{\sigma} \cdot \boldsymbol{n}=(\boldsymbol{I}-\boldsymbol{n} \otimes \boldsymbol{n}) \cdot \boldsymbol{p}+\phi_{0}\left(\nabla_{s} J_{s}\right) / J_{s}^{2}-\nabla_{s} \phi_{0} / J_{s}\end{array}\right.$

$\phi_{0}$ consists of a structural part $\phi_{0}^{\text {stru }}$ and a chemical part $\phi_{0}^{\text {chem }}$. The former is related to the surface strain energy induced by the surface relaxation and the external loading, while the latter originates from the surface dangling-bond energy, i.e.,

$\phi_{0}=\phi_{0}^{\text {stru }}+\phi_{0}^{\text {chem }}$

$\phi_{0}^{\text {stru }}=\frac{E_{b}}{2 \sin \beta} \sum_{i=1}^{2} a_{0 i} \eta_{i}\left\{\begin{array}{l}{\left[3+\left(\lambda_{i}+\lambda_{i} \varepsilon_{s i}\right)^{-m}-3\left(\lambda_{i}+\lambda_{i} \varepsilon_{s i}\right)\right] \times} \\ {\left[\lambda_{i}^{2} \varepsilon_{s i}^{2}+\left(\lambda_{i}-1\right)^{2}+2 \lambda_{i}\left(\lambda_{i}-1\right) \varepsilon_{s i}\right]}\end{array}\right\}$

$\phi_{0}^{\text {chem }}=\phi_{0 b}\left(1-\frac{D_{0}}{w_{1} D}\right), \eta_{1}=a_{01} / a_{02}, \eta_{2}=a_{02} / a_{01}$

where $\phi_{0 b}$ is the surface energy density of the corresponding bulk material. $D_{0}$ is a critical size and we have $D_{0}=3 d_{a}$ for nanoparticles, nanowires and $2 d_{a}$ for nano-films, where $d_{a}$ is the atomic diameter. $D$ denotes a characteristic scale of nanomaterials (e.g., thickness, diameter, etc.). $w_{1}$ is a parameter governing the size-dependent behavior of $\phi_{0}^{\text {chem }}$. $E_{b}$ is the Young's modulus of the corresponding bulk material, which is called as the bulk Young' modulus in the present paper. $a_{01}, a_{02}$ represent the initial lattice lengths in the two principal directions on surface, respectively, as shown in Fig. 1 . The lattice lengths become $a_{r 1}$ and $a_{r 2}$ after spontaneous surface relaxation, and subsequently become $a_{1}$ and $a_{2}$ in the current configuration when subjected to an external loading. $\lambda_{i}=a_{r i} / a_{0 i}$ denotes the surface relaxation parameter, $\varepsilon_{s i}=\left(a_{i}-a_{r i}\right) / a_{r i}$ is the surface strain induced only by the external loading; $m$ is a parameter describing the dependence of bond lengths on the binding energy, where $m=4$ for alloys or compounds and $m=1$ for pure metals. Details can be found in Chen and Yao [37].

One can see that the surface effect of a nano-solid is characterized by two independent parameters in the present theory, i.e., the surface energy density of bulk materials $\phi_{0 b}$ and the surface relaxation parameter $\lambda_{i}$, both of which have clearly physical meanings and are very easy to find through Material Handbooks and simple MD simulations.

\section{Flexural vibration of a Timoshenko nanobeam}

The resonant behavior of a Timoshenko nanobeam with a fixed-fixed or a cantilevered boundary condition is investigated with the above surface energy density-based elastic theory [37]. The beam model is shown in Fig. 2. Consider the nano-sized characteristic length and the small aspect ratio of a Timoshenko beam. Not only the surface effect but also the shear deformation and rotary inertia one on the resonant vibration 


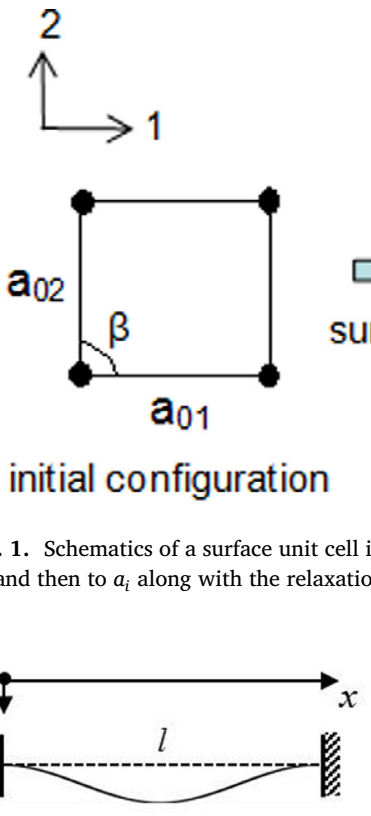

(a)

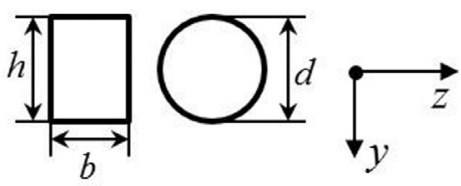

(c)

Fig. 2. Vibration model of a nanobeam in a coordinate system $(x, y, z)$ but with different boundary conditions. (a) A fixed-fixed nanobeam. (b) A cantilevered nanobeam. (c) Two kinds of cross-sections of the nanobeam.

behavior should be included. The length of the nanobeam in the $x$-axis direction is $l$ and the vertical deflection in the $y$-axis direction is denoted as $v$. The cross-section of the nanobeam can be a rectangular shape with a height $h$ and a width $b(b \geq h)$, or a circular one with a diameter $d$ as shown in Fig. 2.

According to the vibration theory of Timoshenko beam [44], when the aspect ratio of a beam is less than about 10 , the cross-section is no longer perpendicular to the longitudinal $x$-axis due to the shear deformation effect. The slope $\partial v / \partial x$ of the deflection curve can be decomposed into a rotation angle $\theta$ due to the pure bending and an additional one $\alpha$ yielded by the shear deformation, i.e.,

$$
\frac{\partial v}{\partial x}=\theta+\alpha
$$

Then, the bending moment $M$ and the shear force $F_{s}$ can be expressed as

$M=-E_{b} I_{z} \frac{\partial \theta}{\partial x}, F_{s}=k G_{b} A \alpha$

where $E_{b}$ is the bulk Young's modulus. $G_{b}=E_{b} /[2(1+v)]$ is the bulk shear modulus and $v$ is the Poisson's ratio. $I_{z}=\int_{A} y^{2} d A$ represents the inertia moment of the cross-section, where $A$ is the area of the crosssection. $k$ denotes a shear coefficient depending on the cross-section shape, where we have $k=5(1+v) /(6+5 v)$ for a rectangular crosssection and $k=6(1+v) /\left(7+12 v+4 v^{2}\right)$ for a circular one.

\subsection{Kinetic equations of a Timoshenko nanobeam}

(a) Based on Eqs. (6) and (7), variation of the bulk strain energy in a vibrating Timoshenko nanobeam yields

$$
\begin{aligned}
\delta \int_{0}^{T} U d t= & -\int_{0}^{T} d t \int_{0}^{l} M \delta\left(\frac{\partial \theta}{\partial x}\right) d x+\int_{0}^{T} d t \int_{0}^{l} F_{s} \delta\left(\frac{\partial v}{\partial x}-\theta\right) d x \\
= & -\int_{0}^{T} d t \int_{0}^{l}\left[E_{b} I_{z} \frac{\partial^{2} \theta}{\partial x^{2}}+k G_{b} A\left(\frac{\partial v}{\partial x}-\theta\right)\right] \delta \theta d x \\
& -\int_{0}^{T} d t \int_{0}^{l} k G_{b} A\left(\frac{\partial^{2} v}{\partial x^{2}}-\frac{\partial \theta}{\partial x}\right) \delta v d x
\end{aligned}
$$

(b) Variation of the surface energy for a vibrating Timoshenko nanobeam can be written as

$\delta \int_{0}^{T} \Phi d t=\int_{0}^{T} d t \int_{S_{n w}} \gamma \cdot \delta \mathbf{u} d S=\int_{0}^{T} d t \int_{0}^{l} d x \int_{C_{n w}}\left(\gamma_{x} \delta u_{x}+\gamma_{n} \delta u_{n}\right) d C$

where $S_{n w}$ and $C_{n w}$ represent the surface area and the perimeter of the nanobeam, respectively. $\delta u_{x}=-y \delta \theta$ and $\delta u_{n} \approx \delta v$ are the horizontal and vertical displacement components of $\delta \mathbf{u}$, respectively. $\gamma_{x}$ and $\gamma_{n}$ represent the tangential and normal components of the surface-induced traction at the nano-beam surface. According to Eq. (2), we have

$\left\{\begin{array}{l}\gamma_{x}=\frac{\partial \phi}{\partial x}=\frac{\partial}{\partial x}\left(\frac{\phi_{0}}{J_{s}}\right)=\frac{1}{J_{s}} \frac{\partial \phi_{0}}{\partial x}-\frac{\phi_{0}}{J_{s}^{2}} \frac{\partial J_{s}}{\partial x} \\ \gamma_{n}=\phi \kappa \approx \frac{\partial^{2} v}{\partial x^{2}} \phi=\frac{\phi_{0}}{J_{s}} \frac{\partial^{2} v}{\partial x^{2}}\end{array}\right.$

where the curvature is $\kappa=-\left(\mathbf{n} \cdot \nabla_{s}\right)=\partial^{2} v / \partial x^{2}$.

The lateral surface of a nanobeam may consist of different crystal facets $[4,12]$. In order to simplify the theoretical analysis, a (100) axially-oriented nanobeam with a symmetric lateral surface is considered, which is similar to the existing literatures $[14,45,46]$. Thus, the surface is perfect and isotropic with an equal atom spacing in both bond directions, e.g., the $(001)$ or $(010)$ surface $[13,30,46]$. Consequently, we have the relaxation parameter $\lambda_{1}=\lambda_{2}=\lambda$ and the lattice length $a_{01}=a_{02}=a_{0}$. The Lagrangian surface energy density of the nanobeam can then be written as [38-40]

$$
\begin{aligned}
& \phi_{0}=\phi_{0 b}\left(1-\frac{3 d_{a}}{4 D}\right)+\frac{\sqrt{2} E_{b} a_{0}}{2}\left[3+\frac{1}{\lambda\left(1+\varepsilon_{x} / 2\right)}-3\left(\lambda+\frac{\lambda \varepsilon_{x}}{2}\right)\right] \\
& \quad \times\left[\frac{\lambda^{2} \varepsilon_{x}^{2}}{4}+2 \lambda(\lambda-1) \frac{\varepsilon_{x}}{2}+(\lambda-1)^{2}\right]
\end{aligned}
$$

Here, $D$ denotes the diameter or height of the nanobeam depending on the shape of the cross section. 
Substituting Eq. (11) into Eq. (10) and using $J_{s}=\lambda^{2}\left(1+\varepsilon_{x} / 2\right)^{2}$ yield the tangential component $\gamma_{x}$ of the surface-induced traction

$\gamma_{x}=\frac{1}{J_{s}} \frac{\partial \phi_{0}}{\partial x}-\frac{\phi_{0}}{J_{s}^{2}} \frac{\partial J_{s}}{\partial x}=\left[C_{0} y+C_{1} y^{2} \frac{\partial \theta}{\partial x}+C_{2} y^{3}\left(\frac{\partial \theta}{\partial x}\right)^{2}\right] \frac{\partial^{2} \theta}{\partial x^{2}}$

$C_{0}=\phi_{0}^{*}(5-4 \lambda)-\frac{\sqrt{2} E_{b} a_{0} A_{2}(3-2 \lambda)}{2}$

$C_{1}=2 \phi_{0}^{*}+\sqrt{2} E_{b} a_{0} A_{1}(3-2 \lambda)-\sqrt{2} E_{b} a_{0} A_{2}(3-2 \lambda)$

$C_{2}=\frac{\sqrt{2} E_{b} a_{0} A_{1}(7-4 \lambda)}{2}-\sqrt{2} E_{b} a_{0} A_{2}$

$\phi_{0}^{*}=\phi_{0 b}\left(1-\frac{3 d_{a}}{4 D}\right)+\frac{\sqrt{2} E_{b} a_{0}}{2}(\lambda-1)^{2}$

$A_{1}=\frac{1-10(\lambda-1)-17(\lambda-1)^{2}}{4}, A_{2}=(\lambda-1)-5(\lambda-1)^{2}$

and the normal one $\gamma_{n}$

$\gamma_{n}=\frac{\phi_{0}}{J_{s}} \frac{\partial^{2} v}{\partial x^{2}}=\left[D_{0}+D_{1} y \frac{\partial \theta}{\partial x}+D_{2} y^{2}\left(\frac{\partial \theta}{\partial x}\right)^{2}\right] \frac{\partial^{2} v}{\partial x^{2}}$

$D_{0}=\phi_{0}^{*}(3-2 \lambda), D_{1}=\phi_{0}^{*}-\frac{\sqrt{2} E_{b} a_{0} A_{2}(3-2 \lambda)}{2}$

$D_{2}=\frac{\sqrt{2} E_{b} a_{0} A_{1}(3-2 \lambda)-\sqrt{2} E_{b} a_{0} A_{2}}{2}$

Then, Eq. (9) can be written as

$\delta \int_{0}^{T} \Phi d t=-\int_{0}^{T} d t \int_{0}^{l}\left\{\begin{array}{l}{\left[C_{0} I_{S 1}+C_{2} I_{S 2}\left(\frac{\partial \theta}{\partial x}\right)^{2}\right] \frac{\partial^{2} \theta}{\partial x^{2}} \delta \theta} \\ +\left[D_{0} I_{C 1}+D_{2} I_{S 1}\left(\frac{\partial \theta}{\partial x}\right)^{2}\right] \frac{\partial^{2} v}{\partial x^{2}} \delta v\end{array}\right\} d x$

where we have $I_{S 1}=\int_{C_{n w}} y^{2} d C, I_{S 2}=\int_{C_{n w}} y^{4} d C, I_{C 1}=-\int_{C_{w w}} n_{w}^{2} d C . n_{w}$ represents the vertical component of the unit normal vector $\boldsymbol{n}$, which is parallel to the deflection $v$.

For different cross-section shapes, we have

Rectangular : $I_{z}=\frac{b h^{3}}{12}, I_{S 1}=\frac{b h^{2}}{2}+\frac{h^{3}}{6}, I_{S 2}=\frac{b h^{4}}{8}+\frac{h^{5}}{40}, I_{C 1}=2 b, A=b h$

Circular $: I_{z}=\frac{\pi d^{4}}{64}, I_{S 1}=\frac{\pi d^{3}}{8}, I_{S 2}=\frac{3 \pi d^{5}}{128}, I_{C 1}=\frac{\pi d}{2}, A=\frac{\pi d^{2}}{4}$

(c) Variation of the kinetic energy of the nanobeam can be written as

$\delta \int_{0}^{T} K d t=\delta \int_{0}^{T} d t \int_{V} \frac{1}{2} \rho\left(\frac{\partial v}{\partial t}\right)^{2} d V+\delta \int_{0}^{T} d t \int_{V} \frac{1}{2} y^{2} \rho\left(\frac{\partial \theta}{\partial t}\right)^{2} d V$

$=-\int_{0}^{T} d t \int_{0}^{l} \rho A \frac{\partial^{2} v}{\partial t^{2}} \delta v d x-\int_{0}^{T} d t \int_{0}^{l} \rho I_{z} \frac{\partial^{2} \theta}{\partial t^{2}} \delta \theta d x$

where $\rho$ denote the material density of the nanobeam.

Combining Eqs. (8), (14) and (16) leads to the variation of the total potential energy,

$$
\begin{aligned}
& \delta \int_{0}^{T}(K-U-\Phi) d t \\
& \quad=\int_{0}^{T} d t \int_{0}^{l}\left[\left(E_{b} I_{z}+C_{0} I_{S 1}\right) \frac{\partial^{2} \theta}{\partial x^{2}}+k G_{b} A\left(\frac{\partial v}{\partial x}-\theta\right)-\rho I_{z} \frac{\partial^{2} \theta}{\partial t^{2}}\right] \delta \theta d x \\
& \quad+\int_{0}^{T} d t \int_{0}^{l}\left[\left(k G_{b} A+D_{0} I_{C 1}\right) \frac{\partial^{2} v}{\partial x^{2}}-k G_{b} A \frac{\partial \theta}{\partial x}-\rho A \frac{\partial^{2} v}{\partial t^{2}}\right] \delta v d x=0
\end{aligned}
$$

with which the kinetic equations of a Timoshenko nanobeam are obtained as

$\left\{\begin{array}{l}\rho I_{z} \frac{\partial^{2} \theta}{\partial t^{2}}=\left(E_{b} I_{z}+C_{0} I_{S 1}\right) \frac{\partial^{2} \theta}{\partial x^{2}}+k G_{b} A\left(\frac{\partial v}{\partial x}-\theta\right) \\ \rho A \frac{\partial^{2} v}{\partial t^{2}}=\left(k G_{b} A+D_{0} I_{C 1}\right) \frac{\partial^{2} v}{\partial x^{2}}-k G_{b} A \frac{\partial \theta}{\partial x}\end{array}\right.$

Let $x=l \hat{x}, v(x, t)=l \hat{v}(\hat{x}, t)$ and $\theta(x, t)=\hat{\theta}(\hat{x}, t)$, Eq. (18) can be rewritten as

$\left\{\begin{array}{l}\chi_{3} \chi_{4} \frac{\partial^{2} \hat{\theta}}{\partial t^{2}}=\left(1+\chi_{1}\right) \chi_{3} \frac{\partial^{2} \hat{\theta}}{\partial \hat{x}^{2}}+\left(\frac{\partial \hat{v}}{\partial \hat{x}}-\hat{\theta}\right) \\ \chi_{3} \chi_{5} \frac{\partial^{2} \hat{v}}{\partial t^{2}}=\left(1+\chi_{2} \chi_{3}\right) \frac{\partial^{2} \hat{\hat{v}}}{\partial \hat{x}^{2}}-\frac{\partial \hat{\theta}}{\partial \hat{x}}\end{array}\right.$

where we have $\chi_{1}=C_{0} I_{S 1} /\left(E_{b} I_{z}\right), \quad \chi_{2}=D_{0} I_{C 1} l^{2} /\left(E_{b} I_{z}\right), \quad \chi_{3}=$ $E_{b} I_{z} /\left(k G_{b} A l^{2}\right), \chi_{4}=\rho I_{z} l^{2} /\left(E_{b} I_{z}\right)$ and $\chi_{5}=\rho A l^{4} /\left(E_{b} I_{z}\right) . \chi_{1}$ and $\chi_{2}$ are two dimensionless parameters characterizing the surface effect of nanobeams. A large enough characteristic size of a nanobeam would induce $\chi_{1}$ and $\chi_{2}$ to approach zero, which can subsequently reduce Eq. (19) to be the classical kinetic equations

$\left\{\begin{array}{l}\chi_{3} \chi_{4} \frac{\partial^{2} \hat{\theta}}{\partial t^{2}}=\chi_{3} \frac{\partial^{2} \hat{\theta}}{\partial \hat{x}^{2}}+\left(\frac{\partial \hat{v}}{\partial \hat{x}}-\hat{\theta}\right) \\ \chi_{3} \chi_{5} \frac{\partial^{2} \hat{v}}{\partial t^{2}}=\frac{\partial^{2} \hat{v}}{\partial \hat{x}^{2}}-\frac{\partial \hat{\theta}}{\partial \hat{x}}\end{array}\right.$

\subsection{Solution of the resonant frequency}

Let $\hat{v}(\hat{x}, t)=\hat{V}(\hat{x}) \cos \omega_{n} t, \hat{\theta}(\hat{x}, t)=\hat{\Theta}(\hat{x}) \cos \omega_{n} t$ and eliminate $\hat{\theta}$ in Eq. (19). We have

$\left\{\begin{array}{l}\left(1+\chi_{1}\right)\left(1+\chi_{2} \chi_{3}\right) \frac{d^{4} \hat{V}}{d \hat{x}^{4}} \\ +\left[\left(1+\chi_{1}\right) \chi_{3} \chi_{5} \omega_{n}^{2}+\chi_{4}\left(1+\chi_{2} \chi_{3}\right) \omega_{n}^{2}-\chi_{2}\right] \frac{d^{2} \hat{V}}{d \hat{x}^{2}} \\ -\chi_{5} \omega_{n}^{2}+\chi_{3} \chi_{4} \chi_{5} \omega_{n}^{4}=0 \\ \frac{d \hat{\Theta}}{d \hat{x}}=\left(1+\chi_{2} \chi_{3}\right) \frac{d^{2} \hat{V}}{d \hat{x}^{2}}+\chi_{3} \chi_{5} \omega_{n}^{2}\end{array}\right.$

where $\hat{V}(\hat{x})$ and $\hat{\Theta}(\hat{x})$ are the dimensionless time-independent mode shape and $\omega_{n}$ is the $n$th angular resonant frequency of the nanobeam.

The general solution of $\hat{V}(\hat{x})$ and $\hat{\Theta}(\hat{x})$ can be expressed as,

$\left\{\begin{array}{l}\hat{V}(\hat{x})=P_{0} e^{\xi \hat{x}} \\ \hat{\Theta}(\hat{x})=Q_{0} e^{\xi \hat{x}}\end{array}\right.$

Substituting Eq. (22) into the first equation in Eq. (21) leads to the following equation

$\xi^{4}+\eta_{1} \xi^{2}+\eta_{2}=0$

where

$\eta_{1}=-\frac{\chi_{2}}{\left(1+\chi_{2} \chi_{3}\right)\left(1+\chi_{1}\right)}+\left[\frac{\chi_{4}}{\left(1+\chi_{1}\right)}+\frac{\chi_{3} \chi_{5}}{\left(1+\chi_{2} \chi_{3}\right)}\right] \omega_{n}^{2}$

$\eta_{2}=\frac{\chi_{3} \chi_{4} \chi_{5}}{\left(1+\chi_{2} \chi_{3}\right)\left(1+\chi_{1}\right)} \omega_{n}^{4}-\frac{\chi_{5}}{\left(1+\chi_{2} \chi_{3}\right)\left(1+\chi_{1}\right)} \omega_{n}^{2}$

Then, the solution of $\hat{V}(\hat{x})$ and $\hat{\Theta}(\hat{x})$ can be written as,

$\hat{V}(\hat{x})=P_{1} \sinh \left(\xi_{1} \hat{x}\right)+P_{2} \cosh \left(\xi_{1} \hat{x}\right)+P_{3} \sin \left(\xi_{2} \hat{x}\right)+P_{4} \cos \left(\xi_{2} \hat{x}\right)$

$\hat{\Theta}(\hat{x})=Q_{1} \sinh \left(\xi_{1} \hat{x}\right)+Q_{2} \cosh \left(\xi_{1} \hat{x}\right)+Q_{3} \sin \left(\xi_{2} \hat{x}\right)+Q_{4} \cos \left(\xi_{2} \hat{x}\right)$

in which

$$
\begin{aligned}
& \xi_{1}=\sqrt{\sqrt{\left(\frac{\eta_{1}}{2}\right)^{2}-\eta_{2}}-\frac{\eta_{1}}{2}}, \xi_{2}=\sqrt{\sqrt{\left(\frac{\eta_{1}}{2}\right)^{2}-\eta_{2}}+\frac{\eta_{1}}{2}} \\
& \text { when } \omega_{n} \leq \sqrt{1 /\left(\chi_{3} \chi_{4}\right)}
\end{aligned}
$$

Substituting Eqs. (25) and (26) into the second equation in Eq. (21) leads to

$\left\{\begin{array}{l}Q_{1}=\zeta_{1} P_{2} \\ Q_{2}=\zeta_{1} P_{1} \\ Q_{3}=-\zeta_{2} P_{4} \\ Q_{4}=\zeta_{2} P_{3}\end{array}\right.$

where $\zeta_{1}=\left[\left(1+\chi_{2} \chi_{3}\right) \xi_{1}^{2}+\chi_{3} \chi_{5} \omega_{n}^{2}\right] / \xi_{1}, \zeta_{2}=\left[\left(1+\chi_{2} \chi_{3}\right) \xi_{2}^{2}-\chi_{3} \chi_{5} \omega_{n}^{2}\right] / \xi_{2}$. $P_{1}, P_{2}, P_{3}$ and $P_{4}$ can be determined by the boundary conditions.

Timoshenko nanobeams with two kinds of boundary conditions are considered. One is a fixed-fixed nanobeam (FF) and the other is a cantilevered one (CA). The respective boundary conditions can be written as

$\left\{\begin{array}{l}\hat{V}(0)=\hat{\Theta}(0)=\hat{V}(1)=\hat{\Theta}(1)=0(F F) \\ \hat{V}(0)=\hat{\Theta}(0)=\frac{d \hat{\Theta}(1)}{d \hat{x}}=\frac{d^{2} \hat{\Theta}(1)}{d \hat{x}^{2}}=0(C A)\end{array}\right.$ 
Table 1

Different material parameters involved or used in this paper $[10,49,50]$.

\begin{tabular}{llllllll}
\hline & $d_{a}(\mathrm{~nm})$ & $a_{0}(\mathrm{~nm})$ & $E_{b}(\mathrm{GPa})$ & $v$ & $\rho\left(\mathrm{kg} / \mathrm{m}^{3}\right)$ & $\phi_{b(100)}(\mathrm{N} / \mathrm{m})$ & $c_{1(100)}(\mathrm{nm})$ \\
\hline $\mathrm{Au}$ & 0.2884 & 0.42 & 79 & 0.44 & 19,300 & 1.63 & 0.025 \\
$\mathrm{Si}$ & 0.22 & 0.54 & 169 & 0.25 & 2330 & 2.2 & - \\
\hline
\end{tabular}

Substituting Eqs. (25) and (26) into Eq. (29) and combining with Eq. (28) lead to the following frequency equations for two kinds of nanobeams, respectively,

$\left|\begin{array}{cccc}0 & 1 & 0 & 1 \\ \zeta_{1} & 0 & \zeta_{2} & 0 \\ \sinh \left(\xi_{1}\right) & \cosh \left(\xi_{1}\right) & \sin \left(\xi_{2}\right) & \cos \left(\xi_{2}\right) \\ \zeta_{1} \cosh \left(\xi_{1}\right) & \zeta_{1} \sinh \left(\xi_{1}\right) & \zeta_{2} \cos \left(\xi_{2}\right) & -\zeta_{2} \sin \left(\xi_{2}\right)\end{array}\right|=0(F F)$

and

$\left|\begin{array}{cccc}0 & 1 & 0 & 1 \\ \zeta_{1} & 0 & \zeta_{2} & 0 \\ \zeta_{1} \xi_{1} \sinh \left(\xi_{1}\right) & \zeta_{1} \xi_{1} \cosh \left(\xi_{1}\right) & -\zeta_{2} \xi_{2} \sin \left(\xi_{2}\right) & -\zeta_{2} \xi_{2} \cos \left(\xi_{2}\right) \\ \zeta_{1} \xi_{1}^{2} \cosh \left(\xi_{1}\right) & \zeta_{1} \xi_{1}^{2} \sinh \left(\xi_{1}\right) & -\zeta_{2} \xi_{2}^{2} \cos \left(\xi_{2}\right) & \zeta_{2} \xi_{2}^{2} \sin \left(\xi_{2}\right)\end{array}\right|=0(C A)$

Solving Eqs. (30) and (31) numerically yield the angular resonant frequency $\omega_{n}$ for the two kinds of nanobeams, which results in the $n$th resonant frequency $f_{n}=\omega_{n} / 2 \pi$.

\section{Results and discussions}

As mentioned earlier, for a Timoshenko nanobeam, not only the surface effect but also the shear deformation effect and the rotary inertia one on the flexural vibration behavior should be included. As a typical example, golden and silicon nanobeams with (100) lateral surfaces are investigated in this paper. According to Diao et al. [47] and Olsson and Park [48], the isotropic surface relaxation parameter $\lambda$ can be empirically expressed as $\lambda=1-c_{1} / D\left(c_{1}>0, D=h\right.$ ord $)$. When the characteristic length $D$ is large enough, $\lambda$ tends to be unity. The value of $c_{1}$ and the other material parameters involved in the following theoretical analysis are listed in Table $1[10,49,50]$.

\subsection{The case of a fixed-fixed nanobeam}

The normalized 1 st resonant frequency $f_{1} / f_{1}^{c}$ as a function of the height $h$ of a fixed-fixed gold (Au) nanobeam is predicted by different elastic models as shown in Fig. 3, including the classical Timoshenko beam model, the Euler-Bernoulli nano-beam model and the Timoshenko nano-beam model. $f_{1}^{c}=4.73^{2} /\left(2 \pi \sqrt{\chi_{5}}\right)$ denotes the 1 st resonant frequency predicted by the classical Euler-Bernoulli beam theory. The numerical results given by Park and Klein [15] are also given for comparison. The length of the rectangular cross-section nanobeam is fixed $l=232 \mathrm{~nm}$ and the width of the cross-section equals the height i.e., $b=h$. The other material parameters of Au are listed in Table 1. It is clearly shown that the theoretical results predicted by both models with surface effect are qualitatively consistent with the numerical ones. The normalized 1 st resonant frequency increases with a decreasing height $h$ not only for an Euler-Bernoulli nanobeam but also for a Timoshenko one. When the height of the nanobeam is relatively small, less than $12 \mathrm{~nm}$ in this case, the results predicted by both the Euler-Bernoulli nanobeam model and the Timoshenko one agree well with each other but deviates significantly from the classical Timoshenko result. It demonstrates that only the surface effect is obvious while the shear deformation effect and the rotary inertia one can be neglected for nanobeams with a relatively small height and a large aspect ratio, i.e., $l / h \geq 19.3$ in this case. When the height of the Au nanobeam is larger than $12 \mathrm{~nm}$, the Timoshenko result

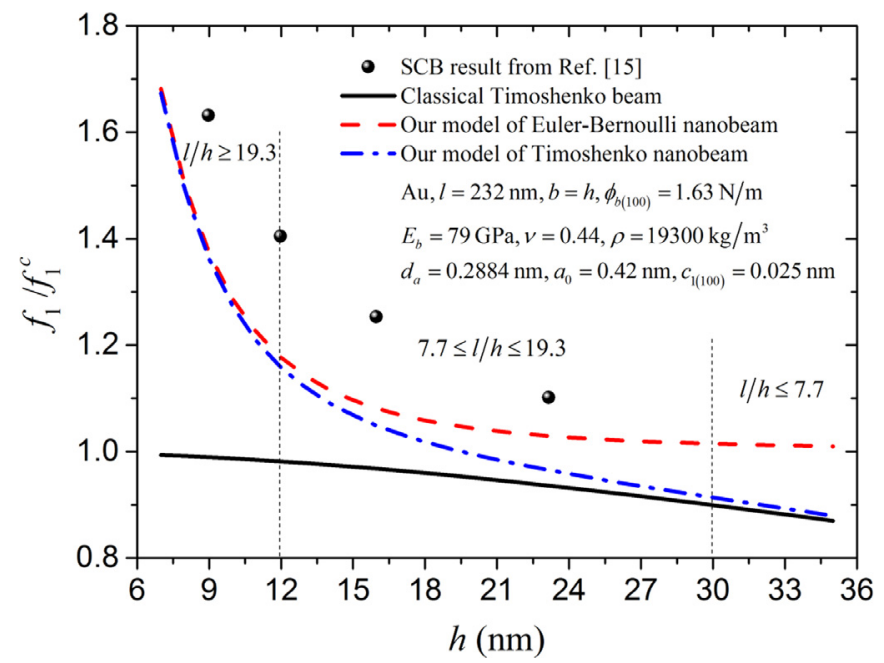

Fig. 3. The normalized 1st resonance frequency predicted by different beam models as a function of the height of a rectangular fixed-fixed gold nanobeam. The corresponding numerical results obtained by Park and Klein [15] is also given for comparison.

with surface effect deviates obviously from the Euler-Bernoulli one and the classical Timoshenko result. It means that not only the surface effect but also the shear deformation one and the rotary inertia one would show significant influence on the vibration behavior of a beam in this region, i.e., an intermediate nano-height and an intermediate aspect ratio, i.e., $7.7 \leq l / h \leq 19.3$ in this case. When the height of the Au nanobeam is beyond about $30 \mathrm{~nm}$, the Timoshenko result including surface effect approaches to the result predicted by the classical Timoshenko beam theory but deviate more significantly from the Euler-Bernoulli result including surface effect. It denotes that the surface effect in this region can be neglected while the shear deformation effect and the rotary inertia one should be considered for nanobeams with a relatively large height and a small aspect ratio, i.e., $l / h \leq 7.7$ in this case.

Comparing the Euler-Bernoulli nanobeam result with the Timoshenko one demonstrates that the shear deformation effect and the rotary inertia one would decrease the resonant frequency of a stubby nanobeam. The 1st resonant frequency predicted by the Timoshenko nanobeam model is reduced in contrast to the resonant frequency $f_{1}^{c}$ predicted by the classical Euler-Bernoulli beam when the aspect ratio is relatively small. However, when the aspect ratio becomes large, i.e., the case with a decreasing height, the 1st resonant frequency of a Timoshenko nanobeam is larger than $f_{1}^{c}$. An interesting fact is that fixedfixed nanobeams would experience a transition from a high frequency to a low one in contrast to the classical Euler-Bernoulli beam result, which is essential a competition result between the surface effect and the shear deformation one and rotary inertia one. The transition point in this case is $h=19.5 \mathrm{~nm}$ or $l / h=11.9$, where the resonant frequency predicted by the Timoshenko nanobeam model equals to that given by the classical Euler-Bernoulli beam model.

\subsection{The case of a cantilevered nanobeam}

The resonant frequency of cantilevered silicon (Si) nanobeams as a function of the nanobeam height is analyzed in this sub-section with three kinds of different models, i.e., the classical Timoshenko beam model, the Euler-Bernoulli nano-beam model and the Timoshenko nano- 


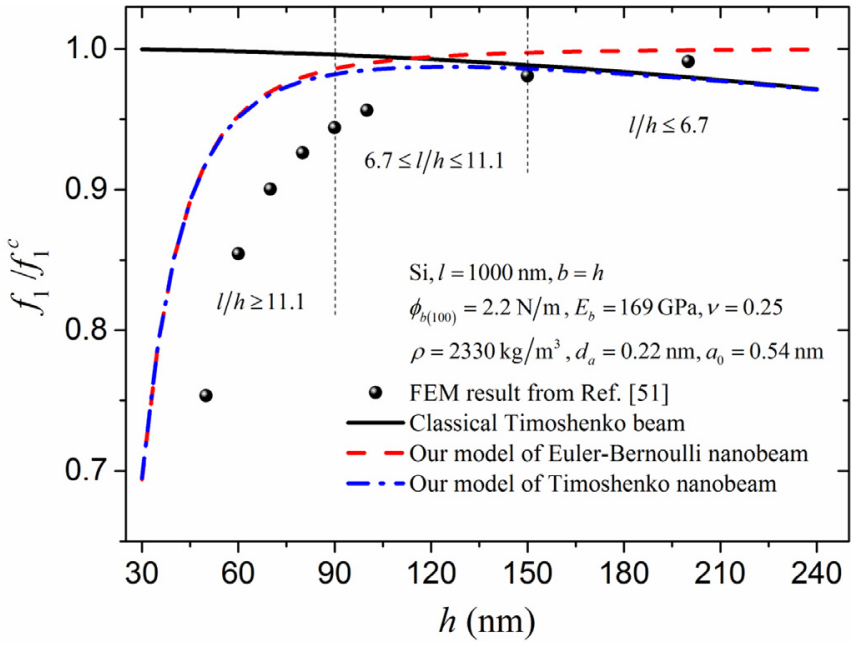

Fig. 4. The normalized 1st resonance frequency predicted by different beam models as a function of the height of a rectangular cantilevered silicon nanobeam. The finite element results obtained by Feng et al. [51] is also given for comparison.

beam model, as shown in Fig. 4, where the finite element result calculated by Feng et al. [51] is also given for comparison. The cross section of the analyzed nanobeam is rectangular with a fixed length $l=1000$ $\mathrm{nm}$ and the height of the nanobeam $h$ varying from $30 \mathrm{~nm}$ to $240 \mathrm{~nm}$. $f_{1}^{c}=1.875^{2} /\left(2 \pi \sqrt{\chi_{5}}\right)$ denotes the 1 st resonant frequency obtained by the classical Euler-Bernoulli cantilevered beam, which is adopted as a normalized parameter in Fig. 4. Generally speaking, the results predicted by the Euler-Bernoulli nano-beam model and the Timoshenko one agree qualitatively with the numerical ones [51]. Comparing with the result predicted by the classical Timoshenko beam model, it is clearly shown that the surface effect would dominate when the height of the cantilevered nanobeam is relatively small (corresponding to a relatively large aspect ratio), i.e., $h \leq 90 \mathrm{~nm}$ or $l / h \geq 11.1$ in this case. While the shear deformation effect and the rotary inertia one would play a dominant role when the height of the cantilevered nanobeam is relatively large (corresponding to a relatively small aspect ratio), i.e., $h \geq 150 \mathrm{~nm}$ or $l / h \leq 6.7$ in this case. In between, not only the surface effect but also the shear deformation effect and the rotary inertia one should be considered, which can be concluded from the comparison among the three kinds of theoretical predictions.

Comparing the normalized resonant frequency of fixed-fixed nanobeams shown in Fig. 3 with that of cantilevered ones shown in Fig. 4 yields that the surface effect would increase the resonant frequency of a fixed-fixed nanobeam while it would decrease the resonant frequency of a cantilever one, in contrast to the results predicted by the classical Timoshenko beam model. It is mainly because the forces in the same direction would cause different directions of curvature in the two structures with different boundary conditions, similar to the phenomenon happening for the effective Young's modulus of nanobeams with different boundary conditions [13-15].

\section{Conclusions}

The dynamic behavior of Timoshenko nanobeams is analyzed by a recently developed elastic theory for nanomaterials. In contrast to the Euler-Bernoulli nanobeams, not only the surface effect induced by the nano-scale characteristic length but also the shear deformation effect as well as the rotary inertia one yielded by in-plane shear stress is considered. The effect of different boundary conditions on the dynamic behaviors of nanobeams is also investigated, including a fixed-fixed case and a cantilevered one. It is found that for nanobeams with a fixed axial length, the surface effect will significantly influence the resonant frequency when the height of the nanobeam is relatively small, i.e., a relatively large aspect ratio; The shear deformation effect as well as the rotary inertia one would show obvious influence on the resonant frequency in contrast to the case of Euler-Bernoulli beam when the height of the nanobeam is relatively larger, i.e., a relatively small aspect ratio; Between the two cases, not only the surface effect but also the shear deformation effect as well as the rotary inertia one would influence the resonant frequency of nanobeams. Furthermore, surface effect would lead to opposite varying trends of resonant frequency for nanobeams with different boundary conditions. In the case of nanobeams with a fixed-fixed boundary condition, surface effect would improve the resonant frequency; while for cantilevered nanobeams, the resonant frequency would be reduced by the surface effect. The main reason is due to the different curvature directions leaded by forces of the same direction. The shear deformation effect and the rotary inertia one would consistently lower the resonant frequency for both fixed-fixed and cantilevered nanobeams. The present results should be helpful for precise design of nanobeam-based nano-devices and useful for accurate evaluation of measured results of nano-instruments.

\section{Acknowledgments}

The work reported here is supported by National Natural Science Foundation of China (No. 11532013, No. 11372317 and No. 11402270), the BIT Creative Research Plan, the Project of Beijing Municipal Science and Technology Commission (No. Z161100001416007) and the Project of State Key Laboratory of Explosion Science and Technology (No. ZDKT17-02).

\section{References}

[1] Rogers JA, Someya T, Huang Y. Materials and mechanics for stretchable electronics. Science 2010;327:1603-7.

[2] Cui Y, Wei QQ, Park H, Lieber CM. Nanowire nanosensors for highly sensitive and selective detection of biological and chemical species. Science 2001;293:1289-92.

[3] Xie P, Xiong QH, Fang Y, Qing Q, Lieber CM. Local electrical potential detection of DNA by nanowire-nanopore sensors. Nat Nanotechnol 2012;7:119-25.

[4] Cuenot S, Frétigny C, Demoustier-Champagne S, Nysten B. Surface tension effect on the mechanical properties of nanomaterials measured by atomic force microscopy. Phys Rev B 2004;69:165410.

[5] Nam CY, Jaroenapibal P, Tham D, Luzzi DE, Evoy S, Fischer JE. Diameter-dependent electromechanical properties of GaN nanowires. Nano Lett 2006;6:153-8.

[6] Gavan KB, Westra HJ, van der Drift EW, Venstra WJ, van der Zant HS. Size-dependent effective Young's modulus of silicon nitride cantilevers. Appl Phys Lett 2009;94:233108.

[7] Li X, Ono T, Wang Y, Esashi M. Ultrathin single-crystalline-silicon cantilever resonators: fabrication technology and significant specimen size effect on Young's modulus. Appl Phys Lett 2003;83:3081-3.

[8] Jing GY, Duan HL, Sun XM, Zhang ZS, Xu J, Li YD, et al. Surface effects on elastic properties of silver nanowires: contact atomic-force microscopy. Phys Rev B 2006;73:235409.

[9] Tan E, Zhu Y, Yu T, Dai L, Sow C, Tan V, et al. Crystallinity and surface effects on Young's modulus of CuO nanowires. Appl Phys Lett 2007;90:163112.

[10] Sadeghian H, Yang CK, Goosen J, Van Der Drift E, Bossche A, French P, et al. Characterizing size-dependent effective elastic modulus of silicon nanocantilevers using electrostatic pull-in instability. Appl Phys Lett 2009;94:221903.

[11] Chen C, Shi Y, Zhang Y, Zhu J, Yan Y. Size dependence of Young's modulus in ZnO nanowires. Phys Rev Lett 2006;96:075505.

[12] Chen Y, Dorgan BL Jr, Mcllroy DN, Aston DE. On the importance of boundary conditions on nanomechanical bending behavior and elastic modulus determination of silver nanowires. J Appl Phys 2006;100:104301.

[13] He J, Lilley CM. Surface effect on the elastic behavior of static bending nanowires. Nano Lett 2008;8:1798-802.

[14] He J, Lilley CM. Surface stress effect on bending resonance of nanowires with different boundary conditions. Appl Phys Lett 2008;93:263108.

[15] Park HS, Klein PA. Surface stress effects on the resonant properties of metal nanowires: the importance of finite deformation kinematics and the impact of the residual surface stress. J Mech Phys Solids 2008;56:3144-66.

[16] Akgöz B, Civalek Ö. A new trigonometric beam model for buckling of strain gradient microbeams. Int J Mech Sci 2014:81:88-94.

[17] Akgöz B, Civalek Ö. A novel microstructure-dependent shear deformable beam model. Int J Mech Sci 2015;99:10-20.

[18] Ma H, Gao X, Reddy J. A microstructure-dependent Timoshenko beam model based on a modified couple stress theory. J Mech Phys Solids 2008;56:3379-91.

[19] Mercan K, Civalek Ö. Buckling analysis of Silicon carbide nanotubes (SiCNTs) with surface effect and nonlocal elasticity using the method of HDQ. Compos Part B 2017;114:34-45. 
[20] Rahmani O, Asemani S, Hosseini S. Study the surface effect on the buckling of nanowires embedded in Winkler-Pasternak elastic medium based on a nonlocal theory. J Nanostruct 2016;6:90-5.

[21] Thai HT. A nonlocal beam theory for bending, buckling, and vibration of nanobeams. Int J Eng Sci 2012;52:56-64.

[22] Wang B, Zhao J, Zhou S. A micro scale Timoshenko beam model based on strain gradient elasticity theory. Eur J Mech A Solids 2010;29:591-9.

[23] Gurtin ME, Murdoch AI. A continuum theory of elastic material surfaces. Arch Ration Mech Anal 1975;57:291-323.

[24] Gurtin ME, Murdoch AI. Surface stress in solids. Int J Solids Struct 1978:14:431-40.

[25] Wang GF, Feng XQ. Effects of surface elasticity and residual surface tension on the natural frequency of microbeams. Appl Phys Lett 2007;90:231904.

[26] Abbasion S, Rafsanjani A, Avazmohammadi R, Farshidianfar A. Free vibration of microscaled Timoshenko beams. Appl Phys Lett 2009;95:143122.

[27] Farshi B, Assadi A, Alinia-Ziazi A. Frequency analysis of nanotubes with consideration of surface effects. Appl Phys Lett 2010;96:093105.

[28] Lee HL, Chang WJ. Surface effects on frequency analysis of nanotubes using nonlocal Timoshenko beam theory. J Appl Phys 2010;108:093503.

[29] Jiang LY, Yan Z. Timoshenko beam model for static bending of nanowires with surface effects. Phys E 2010;42:2274-9.

[30] Wang GF, Feng XQ. Timoshenko beam model for buckling and vibration of nanowires with surface effects. J Phys D Appl Phys 2009;42:155411.

[31] Chen D, Sun D, Li X. Surface effects on resonance frequencies of axially functionally graded Timoshenko nanocantilevers with attached nanoparticle. Compos Struct 2017; 173:116-26.

[32] Chiu MS, Chen T. Effects of high-order surface stress on buckling and resonance behavior of nanowires. Acta Mech 2012;223:1473-84.

[33] Zhang Y, Zhuo LJ, Zhao HS. Determining the effects of surface elasticity and surface stress by measuring the shifts of resonant frequencies. Proc R Soc A 2013;469:20130449.

[34] Miller RE, Shenoy VB. Size-dependent elastic properties of nanosized structural elements. Nanotechnol 2000;11:139.
[35] Mi C, Jun S, Kouris DA, Kim SY. Atomistic calculations of interface elastic properties in noncoherent metallic bilayers. Phys Rev B 2008;77:439-46.

[36] Shenoy VB. Atomistic calculations of elastic properties of metallic fcc crystal surfaces. Phys Rev B 2005;71:094104.

[37] Chen SH, Yao Y. Elastic theory of nanomaterials based on surface-energy density. J Appl Mech 2014;81:121002.

[38] Yao Y, Chen SH. Surface effect on resonant properties of nanowires predicted by an elastic theory for nanomaterials. J Appl Phys 2015;118:044303.

[39] Yao Y, Chen SH. Buckling behavior of nanowires predicted by a new surface energy density model. Acta Mech 2016;227:1799-811.

[40] Yao Y, Chen SH. Surface effect in the bending of nanowires. Mech Mater 2016;100:12-21.

[41] Jia N, Yao Y, Yang YZ, Chen SH. Size effect in the bending of a Timoshenko nanobeam. Acta Mech 2017;228:2363-75.

[42] He Q, Lilley CM. Resonant frequency analysis of Timoshenko nanowires with surface stress for different boundary conditions. J Appl Phys 2012;112:074322.

[43] Yao Y, Chen S, Fang D. An interface energy density-based theory considering the coherent interface effect in nanomaterials. J Mech Phys Solids 2017;99:321-37.

[44] Timoshenko S, Goodier J. Theory of elasticity. New York: McGraw Hill; 1951.

[45] Chhapadia P, Mohammadi P, Sharma P. Curvature-dependent surface energy and implications for nanostructures. J Mech Phys Solids 2011;59:2103-15.

[46] Song F, Huang G, Park H, Liu X. A continuum model for the mechanical behavior of nanowires including surface and surface-induced initial stresses. Int J Solids Struct 2011;48:2154-63.

[47] Diao J, Gall K, Dunn ML. Atomistic simulation of the structure and elastic properties of gold nanowires. J Mech Phys Solids 2004;52:1935-62.

[48] Olsson PA, Park HS. On the importance of surface elastic contributions to the flexural rigidity of nanowires. J Mech Phys Solids 2012;60:2064-83.

[49] Sheng H, Kramer M, Cadien A, Fujita T, Chen M. Highly optimized embedded-atom-method potentials for fourteen fcc metals. Phys Rev B 2011;83:134118.

[50] Jaccodine R. Surface energy of germanium and silicon. J Electrochem Soc 1963;110:524-7.

[51] Feng Y, Liu Y, Wang B. Finite element analysis of resonant properties of silicon nanowires with consideration of surface effects. Acta Mech 2011;217:149-55. 